\title{
Experiencia de enseñar a distancia del profesorado de educación básica
}

\section{Teaching practice experience of basic education teachers in virtual settings}

Norma Guadalupe Márquez Cabellos*

ADRIANA ISABEL ANDRADE SÁNCHEZ**
El confinamiento por el virus SARS Cov-2 ha impulsado al colectivo docente a aprender y adaptarse a nuevas situaciones que impone y demanda la educación a distancia, $y$, por ende, a nuevas formas de enseñar. Esta investigación transversal con alcance descriptivo identifica la experiencia de la práctica de enseñanza del profesorado de educación básica en escenarios virtuales. Participaron 488 docentes, seleccionados de manera no probabilística, por oportunidad, pertenecientes a escuelas de educación preescolar, primaria y secundaria del estado de Colima, México. Se diseñó una encuesta en Google Forms con 23 ítems, que permitió obtener información de la organización y planificación de contenidos curriculares, estrategias implementadas con mediación tecnológica y su impacto en el aprendizaje, estimación del nivel de participación de actividades y tareas del estudiantado, desafíos y experiencia de la práctica docente. Los resultados reflejan variaciones pedagógicas y didácticas en el profesorado; se identifica que las tecnologías digitales han sido esenciales para la implementación de la estrategia de educación a distancia porque facilita el distanciamiento social y el abordaje de los aprendizajes esperados. Se concluye que el profesorado requiere formación, asesoría y acompañamiento para trabajar en formatos de educación con modalidad de enseñanza híbrida.

\section{Palabras clave:}

experiencia docente, educación a distancia, COVID-19, educación básica

Recibido: 29 de junio de 2021 | Aceptado para su publicación: 18 de enero de 2022 |

Publicado: 25 de enero de 2022

Cómo citar: Márquez Cabellos, N. G. y Andrade Sánchez, A. I. (2021). Experiencia de enseñar a distancia del profesorado de educación básica. Sinéctica, Revista Electrónica de Educación, (58), e1336. https://doi.org/10.31391/S2007-7033(2022)0058-004 
Confinement by the SARS Cov-2 virus has prompted the teaching community to learn and adapt to new situations imposed and demanded by distance education, and, therefore, to new ways of teaching. This cross-sectional research with a descriptive scope aimed to identify the teaching practice experience of basic education teachers in virtual settings. 488 teachers who were selected in a non-probabilistic way, by opportunity, belonging to preschool, primary and secondary schools of the state of Colima, México participated. A survey was designed in Google Forms with 23 items that allowed obtaining information on the organization and planning of curricular content, strategies implemented with technological mediation and their impact on learning, estimation of the level of participation in activities and tasks of the student body, challenges, and experience of teaching practice. The results reflect pedagogical and didactic variations in the teaching staff, it is identified that the use of digital technologies has been essential for the implementation of the distance education strategy because it facilitates social distancing and enables the approach of expected learning. It is concluded that teachers require training, advice, and support to work in educational formats with a hybrid teaching modality.

\section{Keywords:}

teaching experience, distance education, COVID-19, basic education

\footnotetext{
*Doctora en Psicología Educativa por la Universidad Autónoma de Aguascalientes, México. Profesora-investigadora de la Facultad de Ciencias de la Educación de la Universidad de Colima. Miembro del Sistema Nacional de Investigadores. Líneas de investigación: atención a las aptitudes sobresalientes, talentos específicos y educación inclusiva. Correo electrónico: norma_marquez@ucol.mx/ https://orcid.org/0000-0001-5466-2681

** Doctora en Estadística Multivariante Aplicada por la Universidad de Salamanca, España. Profesora en la Facultad de Ciencias de la Educación de la Universidad de Colima. Miembro del Sistema Nacional de Investigadores. Línea de investigación: aplicación de métodos estadísticos en ciencias sociales. Correo electrónico: isa_andrade@ucol.mx/ https:// orcid.org/0000-0001-9790-5719
} 


\section{INTRODUCCIÓN}

T a repentina aparición en China del virus denominado SARS Cov-2 a finales de 2019 y su posterior expansión en el mundo en los meses siguientes han representado un reto en los ámbitos de la vida social e individual, en el educativo, político, económico y laboral. Sin duda, el campo educativo se vio afectado severamente por la suspensión de las actividades académicas de más de 1,215 millones de estudiantes de todos los niveles educativos (Unesco, 2020), lo que obligó a efectuar transformaciones pedagógicas radicales en el funcionamiento cotidiano y en las dinámicas en el proceso de enseñanza-aprendizaje y abrió paso a escenarios con el uso de nuevas tecnologías.

Debido a la suspensión de las clases presenciales, diversos países han implementado variedad de estrategias para sostener actividades educativas con mediación tecnológica, y han hecho uso de plataformas virtuales con ajustes razonables en contenidos educativos simplificados, objetivos o metodología. Lamentablemente, el confinamiento por la COVID-19 expuso de forma clara la desigualdad social en las familias en virtud de que la educación en línea solo ha sido posible para quienes tienen acceso a internet y a dispositivos tecnológicos. La Comisión Económica de América Latina (CEPAL, 2020) puntualiza que el $46 \%$ de los niños y niñas entre 5 y 12 años de América Latina viven en hogares que no están conectados a internet, lo que implica la exclusión de más de 32 millones de niños y niñas.

En México, el acceso de los hogares a los dispositivos digitales es también desigual, en especial entre los distintos niveles socioeconómicos y culturales; se identifica una marcada diferencia entre los estratos económicos más altos y más bajos, lo que condiciona el derecho a la educación. Tales diferencias son una pieza más del mosaico de las desigualdades en México; por ello, la carencia en el acceso a la tecnología afecta a estudiantes desde el nivel preescolar hasta educación superior.

Al cerrar sus puertas las escuelas de México a mediados de marzo de 2020, el profesorado intervino pedagógicamente con la población estudiantil con mínima o nula asesoría, capacitación o acompañamiento en el manejo de algunas herramientas tecnológicas, sin formación técnica y didáctica en la implementación de la estrategia académica de educación a distancia. Sin duda, a casi dos años de confinamiento se extraña las aulas, la interacción de estudiantes con toda la comunidad educativa, las prácticas pedagógicas y las reflexiones que de ellas se derivan. En este confinamiento, el profesorado se ha enfrentado a la necesidad de aprender mecanismos técnicos de la educación virtual, y se ha adaptado a nuevas situaciones que impone y demanda la educación a distancia; se ha dejado de lado la premisa que la garantía, la pertinencia y la calidad de la enseñanza en el salón de clases se apoya en la interacción social entre el profesorado y el alumando.

Frente a este hecho histórico, en México, la Secretaría de Educación Pública (SEP) propuso, en abril de 2020, la estrategia de educación a distancia "Aprende en Casa" para educación básica, la cual, a la fecha, tiene tres ediciones: la primera, comprendida de abril a julio de 2020; la segunda, de agosto a diciembre de 2020; y la tercera, de enero a julio de 2021. Con estas, las instituciones educativas acudieron a una diversidad de plataformas tecnológicas en un intento por seguir brindando educación a más de 36 millones de niños y adultos en el país. 
A pesar de la variedad de apoyos educativos de esta estrategia, el Consejo Nacional de Evaluación de la Política de Desarrollo Social (Coneval), en su informe de evaluación de septiembre de 2021, puntualiza como principales hallazgos la carga excesiva de trabajo de directivos y docentes con la modalidad a distancia para mantener comunicación permanente con estudiantes y sus familias, la falta de diseño metodológico de la estrategia, que provoca ajustes durante su operación, la ausencia de indicadores para monitorear el desempeño y resultados de la estrategia, así como la inexistencia de una matriz de indicadores para resultados que verifique los bienes y servicios brindados en la estrategia (Coneval, 2021).

A lo largo del tiempo, la escuela se ha consolidado a través de los sistemas educativos por ser un contexto facilitador y de reconocimiento que promueve oportunidades de participación y aprendizaje, que sirven de soporte y estructura para la inclusión posterior en una sociedad (Valcarce, 2011). Sin embargo, ante este hecho inédito de la pérdida del espacio escolar y del aula, la escuela ha modificado su forma de enseñar y el estudiantado su manera de aprender, sus intereses y habilidades, porque la escuela de nuestros días se ha quedado atrapada en el formalismo del currículo al presentar programas digitales y de televisión alineados con los contenidos académicos, la cumplimentación de la densidad de actividades y tareas, o bien, en el ritual de comunicación con los padres de familia, y ha dejado de lado la oportunidad de educar y formar a la distancia; es decir, acercar la escuela a la vida real del estudiante en la que identifique, razone, argumente, indague o discuta la diversidad de situaciones con las que se enfrenta.

Díaz-Barriga (2020) refiere que "no aprovechar la oportunidad para repensarse, mantiene una visión rígida de la escuela y del currículo, sobre todo para la educación básica" (p. 26). Esta realidad mundial que vivimos nos invita a pensar cómo cosechar esta situación para impulsar otras formas de enseñar, otro tipo de aprendizajes y otro modo de aprender. Aquí radica la importancia que la escuela se active, se reconstruya y esté en estado de alerta permanente por la modalidad híbrida que se brinda en las escuelas debido a la asistencia escalonada de la población estudiantil en las aulas. Consideramos que esta modalidad tiene implicaciones técnicas y pedagógicas para su implementación, porque requiere renovar la enseñanza, no solo para planificar los aprendizajes que se espera adquieran los estudiantes, sino en la didáctica, en el cómo mediar los contenidos en sesiones presenciales y a distancia, así como la diversidad de estrategias metodológicas que debe aplicar en su práctica docente.

Ante el panorama de la estrategia de educación a distancia por la contingencia sanitaria de la COVID-19, nuestro estudio tiene como objetivo identificar la experiencia de la práctica de enseñanza del profesorado de educación básica en escenarios virtuales en torno a la planeación curricular, estrategias implementadas, desafíos presentados, además de la estimación del nivel de comunicación y participación de la población estudiantil y la familia.

Para el estudio, hicimos una búsqueda de literatura relacionada con experiencia docente con mediación tecnológica por el confinamiento de la COVID-19. Encontramos que los estudios estaban enfocados a la evolución e impacto psicológico en población infantil, aprendizajes de estudiantes en escenarios virtuales, así como efectos en el estado emocional y social por el confinamiento (Aguilar, 2020; Albalá y Guido, 2020; Espada et al., 2020; Domínguez et al., 2020; Hernández, Prada y Hernández, 2021). Identificamos un estudio realizado por el 
Centro de Investigación Avanzada en Educación, de la Universidad de Chile, y el Centro de Investigación para la Educación Inclusiva, de la Pontificia Universidad Católica de Valparaíso (2020), que recoge la voz de 2,205 docentes de establecimientos aducacionales sobre la experiencia docente en situación escolar no presencial; en este encontramos cambios en el modo de enseñar y aprender, readecuaciones en los métodos de enseñanza y la comunicación pedagógica con la población estudiantil. Por ello, el vacío presentado hasta el momento para recuperar la experiencia docente en escenarios virtuales hace necesario el estudio.

\section{Metodología}

\section{Participantes}

En esta investigación mixta, transversal con alcance descriptivo (Hernández, Fernández y Baptista, 2014), participaron 488 docentes de educación básica -preescolar, primaria y secundaria-, de los cuales el 65.5\% eran mujeres y el 34.5\%, hombres. Estos participantes se seleccionaron de manera no probabilística, por oportunidad, los cuales pertenecían a escuelas regulares de educación básica del estado de Colima, México.

\section{Instrumento}

A través de una encuesta en Google Forms con 23 ítems (18 ítems con diferentes escalas de respuesta y 5 preguntas abiertas), obtuvimos información de variables sociodemográficas, organización y planificación de contenidos curriculares, estrategias implementadas con mediación tecnológica y su impacto en el aprendizaje, estimación del nivel de participación de actividades y tareas del estudiantado, desafíos de la práctica docente y la experiencia en la práctica de enseñanza.

\section{Procedimiento}

Gestionamos ante la Secretaría de Educación del estado de Colima el permiso para acceder a las personas participantes de este estudio, quienes trabajaban en escuelas públicas de educación básica. Una vez obtenido el permiso, solicitamos el consentimiento informado y voluntario del profesorado participante y especificamos como criterio de inclusión que fueran docentes frente a grupo de educación básica (preescolar, primaria o secundaria). Otorgamos quince días para la cumplimentación de la encuesta en línea y, con la finalidad de proteger el anonimato, no solicitamos ningún dato personal de identificación a los participantes. Para el análisis estadístico de las respuestas, utilizamos porcentajes; las respuestas a las preguntas abiertas se examinaron en forma descriptiva y mediante codificación temática con el programa MaxQda Plus 2020.

\section{RESULTADOS}

Para obtener una perspectiva general de los resultados de los 488 docentes participantes en la investigación, debemos señalar que las edades comprendidas oscilaron entre 27 y 52 años $(M=39.03$; DT= 5.54). Del total de los participantes en el 
estudio, el 65.2\% ( $n=320)$ son docentes de educación primaria, el 20.6\% ( $n=101)$, de educación preescolar, y el 11.8\% (58), de educación secundaria. La mayoría del profesorado (86.6\%) estaba casado o convivía en pareja; el resto (13.4\%) tenía estado civil soltero. El 93\% cursó una licenciatura sobre la docencia, el 7\% egresó de la escuela normal de maestros sin estudios de educación superior, y el 33\% estudió un posgrado relacionado con la educación. En cuanto a su capacitación o actualización académica, el 51\% carece de entrenamiento para enseñar con mediación tecnológica, el $13.6 \%$ ha recibido una actualización muy escasa y el 35.4\% está entrenado con herramientas virtuales.

\section{Organización y planificación de contenidos curriculares}

La mayor parte del profesorado (89\%) que respondió a la encuesta indicó que la organización y estructura de contenidos curriculares se discutió en el marco del Consejo Técnico Escolar y se determinó realizar ajustes razonables en los aprendizajes esperados, propósitos y en las actividades propuestas con mediación tecnológica; el 11\% de los participantes refirieron que la organizaron de la estructura de los aprendizajes curriculares estuvo a su cargo conforme al plan de estudios vigente de educación básica.

El 53.8\% (n=264) de los participantes puntualizaron que en la planeación consideran los aprendizajes esperados de todas las asignaturas correspondientes del grado y nivel educativo a su cargo; el $13.4 \%(n=66)$ priorizan los aprendizajes de la educación socioemocional; el 13.2\% ( $\mathrm{n}=65)$, de español; el 2.9\% $(\mathrm{n}=14)$, de matemáticas; el 2.2\% ( $n=11)$, los contenidos de vida saludable; el $1 \%(n=5)$, de formación cívica y ética; el $0.6 \%(n=3)$, de tecnología; y el $0.2 \%(n=1)$, la asignatura de inglés (ver gráfica 1).

Gráfica 1. Planeación de aprendizajes esperados de las asignaturas del plan de estudios de educación básica

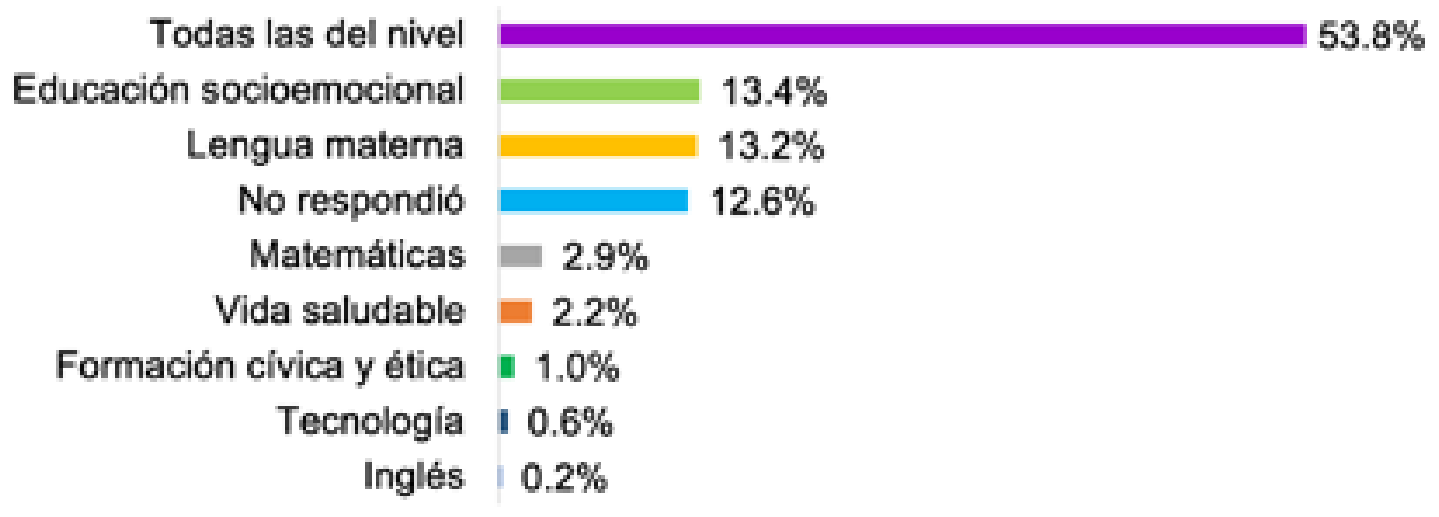

Considerando la compleja situación de la contingencia sanitaria, la comunidad directiva y docente organizó brigadas para la entrega de los libros de texto gratuito a la población estudiantil al inicio del ciclo escolar 2020-2021. Los resultados de la encuesta arrojaron que el $56 \%(n=275)$ de participantes señalaron que más del $90 \%$ de los libros de texto fueron entregados a sus estudiantes; el $17 \%(n=82)$ de profesores y profesoras, que entre el 70\% y el $89 \%$ de los libros de texto; el $7 \%(n=36)$, que menos del $69 \%$ de los libros fueron facilitados; y el resto $(19 \%, n=95)$ no respondió. 
En cuanto a la planificación de las actividades de enseñanza-aprendizaje que implementó el personal docente de educación básica con el apoyo de los libros de texto gratuito, el $80 \%$ refirió que fueron programadas semanalmente promoviendo el trabajo con ese recurso; el 10\% consideró de manera parcial algunas actividades de los libros de texto; y el $10 \%$ no contestó.

Con la estrategia de educación a distancia "Aprende en Casa II", el gobierno mexicano, con la coordinación de la SEP, diseñó la producción del programa televisivo que conforma el contenido educativo específico como apoyo al aprendizaje del alumnado en casa. La SEP determinó esos programas como la base del trabajo educativo durante la pandemia; ante esta premisa, se preguntó al profesorado sobre la promoción del uso del servicio televisivo y se obtuvo que el 31\% de los docentes $(\mathrm{n}=149)$ han promovido su uso en menos del $69 \%$ de sus sesiones; $27 \%$ (129 profesores), entre el $70 \%$ y el $89 \%$ de las veces; $32 \%$ ( $n=156)$, entre el $90 \%$ y el $100 \%$; el $11 \%$ restante no respondió a esta pregunta (ver gráfica 2 ).

Gráfica 2. Promoción del uso del servicio televisivo para fortalecer el aprendizaje

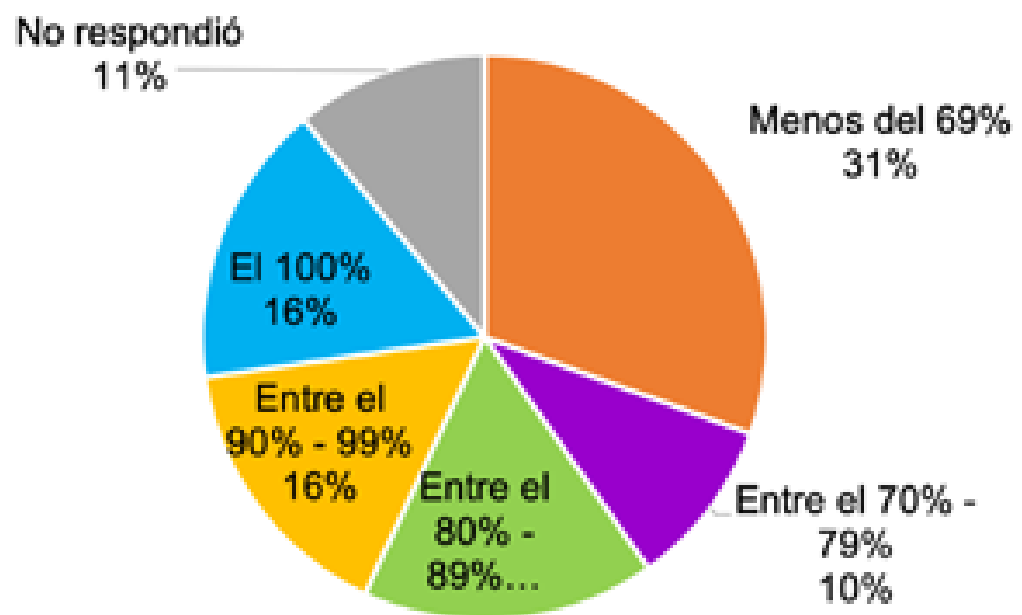

Asimismo, se preguntó a los docentes participantes qué era lo más importante de los programas televisivo de Aprende en Casa II y III, el 48\% señalaron que lo más importante es que abordan los aprendizajes esperados del grado y nivel educativo; el 28\%, que la claridad y pertinencia en las explicaciones de los especialistas que abordan los contenidos académicos; el 10\%, que el colorido y la variedad de recursos didácticos que utilizan; y el 14\% no respondieron.

Estrategias implementadas con mediación tecnológica y su impacto en el aprendizaje El 100\% del profesorado indicó estar ejerciendo su práctica pedagógica a distancia; ninguno ha experimentado enseñanza híbrida durante la contingencia sanitaria por la COVID-19. Con la finalidad de garantizar el aprendizaje en el alumnado, además de las actividades propuestas en los libros de texto gratuito y la barra de contenidos en el programa televisivo Aprende en Casa, el 44\% de docentes participantes han diseñado y entregado cuadernillos de apoyo de lectura-escritura y pensamiento matemático para quienes están en situación de vulnerabilidad por condición de discapacidad o trastorno, que no tienen acceso a internet o no cuentan con herramientas 
tecnológicas; el 22\% elabora videos y audios educativos; el 10\% se apoya en videos educativos de YouTube; el 5\% se auxilia en la plataforma de Google Classroom para promover las actividades de los aprendizaje esperados; el 2\% promueve y soporta su enseñanza en el cuadernillo titulado Vamos de regreso a clases, impulsado por la SEP; el 14\% refiere otros tipos de recursos; y el 4\% no contesó.

Gráfica 3. Estrategias didácticas complementarias que el profesorado implementa

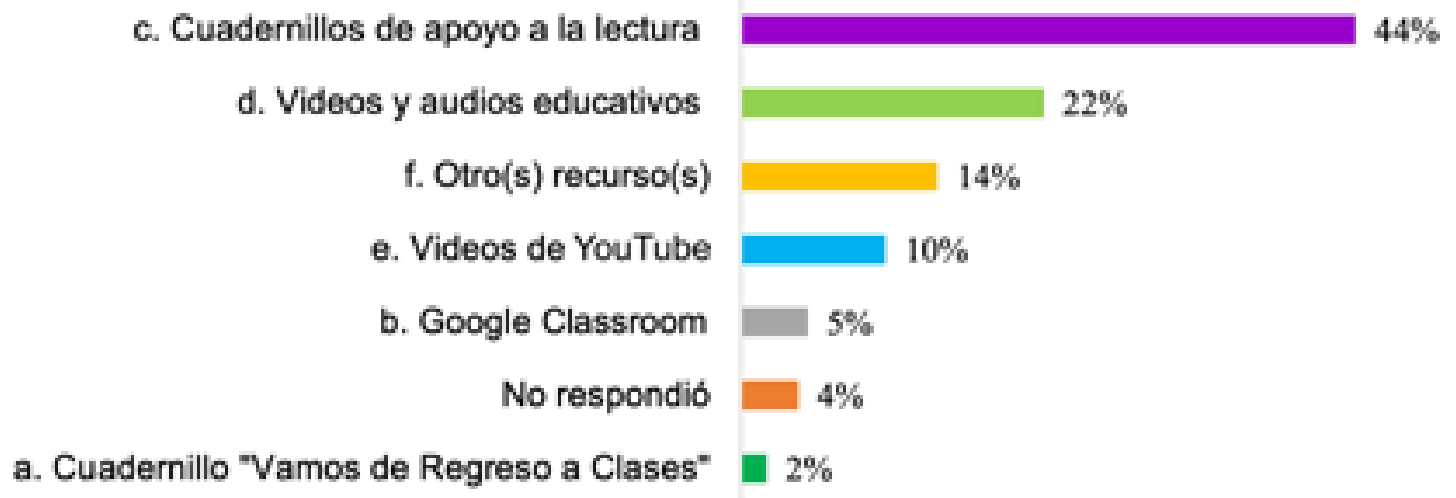

En cuanto a la pregunta abierta sobre el principal avance en el aprendizaje que ha tenido el grupo a su cargo con las estrategias implementadas con mediación tecnológica, la población docente dio respuestas concernientes a los aprendizajes esperados del plan de estudios vigente, el aprendizaje no formal adquirido en casa, así como aquellos que no logran identificar qué avances tiene el alumnado en sus aprendizajes por la falta de comunicación y participación. A continuación algunas respuestas:

... cada uno de mis alumnos tienen realidades muy distintas y por tanto aprendizajes distintos [...]; si los padres de familia se comprometen con su hijo, avanza académicamente, es decir, logra los aprendizajes esperados; de lo contrario, hay un rezago educativo significativo (docente de tercer grado de primaria).

Considero que mis alumnos han avanzado en sus aprendizajes, se ve en las actividades que me entregan del cuadernillo de trabajo y cuando tengo sesión virtual con ellos; han consolidado o están en proceso de consolidar contenidos del campo de lenguaje y comunicación, así como del pensamiento matemático, además que manejan la tecnología muy bien (docente de quinto grado de primaria).

... creo que han aprendido a sobrevivir en esta pandemia más que aprendizajes académicos, porque ya están más grandes y ellos han tenido que colaborar con su familia en las tareas de casa porque sus padres tienen que salir a trabajar [...]; han aprendido a resolver problemas como hacer comida, cuidar de sus hermanos, cuidarse ellos mismos e incluso muchos de ellos a trabajar fuera de casa (docente de tercer grado de secundaria).

Es dificil saber qué tanto han avanzado mis alumnos con esta estrategia de educación a distancia, pues evaluarlos a través de una computadora no sabes si la familia los está apoyando para responder o ellos estan respondiendo solos (docente de segundo de preescolar).

Identifico una variedad de aprendizajes en mis alumnos, aprenden aquellos que sí cumplen; aquellos que más o menos cumplen es evidente que no están avanzando como quisiera y aquellos que he perdido comunicación con sus padres, pues tienen un rezago educativo impresionante porque no sé de ellos (docente de sexto grado de primaria). 


\section{Participación y cumplimiento en actividades y tareas}

Con referencia a esta categoría de análisis relacionada con la participación y la cumplimentación de actividades y tareas por parte del alumnado, el 13\% del profesorado consideró que los padres y las madres de familia colaboran en la realización de tareas y con la conectividad en las sesiones sincrónicas programadas; el 68\% de docentes participantes respondieron que la familia se involucra intermitentemente con la educación a distancia, es decir, envían tareas o conectan a sus hijos en ocasiones; el 19\% de la población participante mencionó que perdieron comunicación con los padres y madres de familia y, por ende, sus estudiantes no envían tareas y no se conectan a las sesiones de trabajo. Algunas evidencias se reflejan en las respuestas siguientes:

Las madres de familia se han mostrado comprometidas con la educación de sus hijos, apoyan en las actividades y envian evidencias [...]; eso me da mucho gusto y certeza que están aprendiendo mis alumnos (docente de cuarto grado de primaria).

... me da gusto cuando recibo las actividades de mis alumnos, el cuadernillo mensual con las actividades realizadas [...], qué bonito sería que todos cumplieran porque se garantizaría el aprendizaje (docente de tercer grado de preescolar).

Es triste e incómodo estar insistiendo a los padres de familia para que sus hijos cumplan con las tareas [...], algunos de ellos hasta me han bloqueado de su celular [...]; tengo varios alumnos con esta situación que no cumplen con las actividades, los padres de familia o quienes están a su cargo no los hacen responsables (docente de segundo grado de secundaria).

Se requiere mayor compromiso por parte de los padres de familia en el acompañamiento de las tareas y actividades con los niños [...]; se entiende la carga de trabajo que originó la pandemia, pero nosotros también estamos haciendo un gran esfuerzo por planear y lograr que el alumno no tenga rezago escolar (docente de cuarto grado de primaria).

Con referencia al ítem relacionado con la comunicación que tiene el profesorado con los padres y madres de familia para coordinar las acciones del proceso de aprendizaje con sus hijos, el $81.6 \%$ menciona que mantiene comunicación permanente con la familia, la cual no garantiza el cumplimiento de las actividades o tareas; el $18.4 \%$ refiere que ha perdido comunicación con los padres de familia sin poder localizarlos y desconoce las causas:

Al inicio de ciclo escolar no se tenía una comunicación constante con la familia [...]; al término del primer trimestre se avanzó tanto en la comunicación con padres de familia como en el aprendizaje de mis alumnos (docente de primer grado de primaria).

Establecer dinámicas de trabajo y un canal de comunicación constante con los padres de familia para organizar y dar seguimiento al trabajo realizado ha sido la clave para que mis alumnos sigan aprendiendo con esta estrategia a distancia (docente de tercer grado de preescolar).

He mantenido buena comunicación con la mayoría de los padres de familia; sin embargo, esto no ha garantizado que se cumpla con las tareas y sugerencias ofrecidas de mi parte [...], es decir, tener comunicación con ellos no garantiza el aprendizaje porque te dicen que si lo harán, pero sus hijos no se comprometen y no envían nada (docente de primer grado de secundaria).

Esta estrategia la hemos padecido mucho las familias, los alumnos y nosotros como docentes, porque hemos insistido con las actividades y tareas, pero los padres de familia están ocupados con su trabajo o a veces mienten porque no cumplen, por ello es que no se logra ver avance en el aprendizaje en los alumnos [...]; a ver cómo nos va cuando regresemos a las aulas (docente de quinto grado de primaria). 


\section{Desafíos en la práctica docente}

Para realizar una evaluación oportuna y eficiente del logro educativo del estudiantado, los avances en los aprendizajes esperados, los niveles de accesibilidad a dispositivos electrónicos, la conectividad y el acompañamiento en las tareas y actividades, la SEP propuso identificar el vínculo de comunicación con madres, padres de familia y tutores; para ello, determinó tres niveles de comunicación y participación de la población estudiantil: sostenida, intermitente e inexistente. A la luz de los resultados obtenidos en este set de ítems, el profesorado participante refiere que el $19.3 \%$ se ubican en el nivel de comunicación inexistente, el 69.1\%, en comunicación intermitente, y el 11.6\%, en comunicación sostenida.

El principal desafío que ha enfrentado el profesorado es el momento de ejercer los criterios de evaluación trimestral al no tener insumos como la cumplimentación de actividades y tareas, y la presencia en actividades sincrónicas por parte del alumnado cuando su comunicación es intermitente (21\%) e inexistente (42\%). Otro desafío que ha tenido que sortear el profesorado es conocer y dominar las orientaciones pedagógicas y los criterios de evaluación (11\%) propuestos por la SEP, debido a que estos son ambiguos y subjetivos (ver gráfica 4).

Gráfica 4. Principal desafío para ejercer los criterios de evaluación

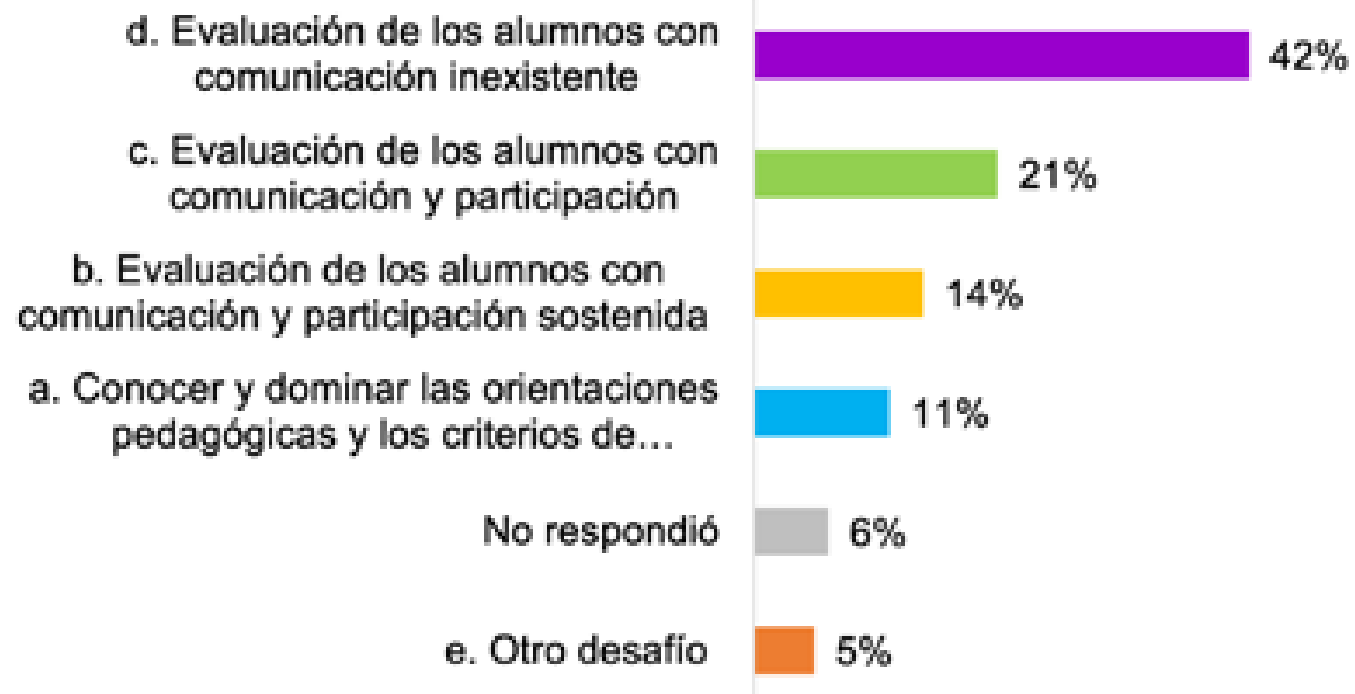

Otros desafíos docentes identificados son aquellos centrandos en la entrega de calificaciones trimestrales de la población estudiantil, pues el 56\% de los padres no muestran la disposición y el tiempo para recibirlo por parte de los docentes; el $28 \%$ presenta dificultades en la conectividad para la asistencia de clases sincrónicas y la elaboración de actividades asincrónicas; el 9\% refleja que las madres, padres y tutores no tienen tiempo para acompañar las actividades académicas de sus hijos; y el 7\% refiere otros desafíos sin puntualizar cuáles. 


\section{Experiencia de la práctica de enseñanza con mediación tecnológica}

Respondiendo al set de preguntas abiertas, le preguntamos al profesorado participante sobre su experiencia docente con la mediación tecnológica e identificamos un mosaico de sentimientos e incertidumbre que, a más de un año, siguen prevaleciendo. Las siguiente son algunas de sus respuestas:

He aprendido mucho cómo trabajar a distancia con diferentes plataformas [...] me he visto en la necesidad de capacitarme porque desconocía el mundo de la tecnología (docente de segundo grado de secundaria).

Mi experiencia ha tenido muchos matices desde la tristeza de no realizar mi práctica docente en el aula, con mis materiales y mis alumnos, hasta la impotencia y molestia de que muchos padres de familia no se involucran con la educación de sus hijos sin identificar que los perjudicados son los alumnos porque no están aprendiendo (docente de sexto grado de primaria).

... ha sido de mucho esfuerzo, tiempo invertido en la capacitación, estar muchas horas frente a mi computadora para preparar las clases, hacer videos, cuadernillos de trabajo, comunicarme con los padres de familia [...]; lo más gratificante y la mejor experiencia es cuando tengo clases virtuales con mis niños (docente de tercero de preescolar).

No ha sido sencilla esta experiencia porque no manejaba nada de las TIC's, sabía lo mínimo [...]; sin embargo, hoy me siento una experta porque he tenido que esforzarme para que mis alumnos aprendan, es decir, facilitarles el aprendizaje (docente de primer grado de secundaria).

Creo que si hubieramos tenido un acompañamiento y claridad de la estrategia de educación a distancia por parte de nuestras autoridades otra cosa hubiera sido, hemos aprendido a punta de tropiezos; esto también ha provocado que los padres de familia y alumnos pierdan el interés y no vean con seriedad la escuela (docente de tercer grado de primaria).

Mi experiencia ha sido de aprendizaje constante por tantos cursos y capacitaciones que he tomado; sin embargo, la mejor experiencia es ver la cara de mis alumnos cuando voy a sus casas a recoger el cuadernillo de trabajo y dejarles el nuevo [...]; esto me hace olvidar todo el cansancio del trabajo extra que realizo para que ellos aprendan (docente de segundo grado de primaria).

... mi mejor experiencia es cuando escucho la voz o veo a mis alumnos a través de una videollamada, cuando me responden acertadamente a lo que pregunto de las actividades escolares (docente de sexto grado de primaria).

\section{CONCLUSIONES Y REFLEXIONES}

A más de un año del confinamiento por la contingencia sanitaria, las formas de enseñar han implicado adecuaciones significativas no solo en la simplificación de aprendizajes esperados del plan de estudio vigente de educación básica, sino en la relación del profesorado con la población estudiantil y los padres de familia o tutores. Toda la comunidad escolar ha tenido que explorar y poner a prueba nuevos modos de enseñanzar y aprender, lo que ha producido un crecimiento personal y social.

Los hallazgos de la investigación revelan que el uso de las tecnologías digitales cobró mayor vida, pues han sido esenciales para la implementación de la estrategia de educación a distancia, porque facilita el distanciamiento social y posibilita parcialmente el abordaje de los aprendizajes esperados. Sin embargo, esta estrategia, integrada por una serie de recursos y materiales transmitidos por televisión y en línea, 
se ancla cuando las familias no tienen acceso y uso de tecnologías de la información; por ello, la comunidad docente se ha visto en la necesidad de recurrir a diversas estrategias, como cuadernillos de actividades, videollamadas, visitas domiciliarias o llamadas telefónicas, para abordar los aprendizajes esperados en la población en situación de vulnerabilidad.

Los resultados de la investigación reflejan también variaciones pedagógicas y didácticas en el profesorado: identificamos el emprendimiento de acciones distintas a las señaladas por la autoridad educativa con el único objetivo de dar continuidad al trabajo que demanda el plan de estudios con o sin el recurso tecnológico y la conectividad de internet en los hogares. Asimismo, el profesorado, en muchas ocasiones, ha dejado de lado la estrategia propuesta por el gobierno federal, lo que ha impulsado la búsqueda constante de alternativas para el logro de la participación y el aprendizaje del alumnado a su cargo.

Un dato significativo es la comunicación intermitente e inexistente que las madres y padres de familia tienen con el profesorado para dar continuidad al aprendizaje de sus hijos, pues los resultados evidencian la baja participación en el acompañamiento académico y la cumplimentación de actividades. Esto nos lleva a deducir que la sobrecarga que implica el hogar, el trabajo, el compromiso social y personal desembocó tensiones, dilemas, estrés, apatía o desinterés por la escuela. A pesar de estos escenarios, el profesorado manifiesta que la enseñanza no se ha detenido, ya que, en mayor o menor medida, la población estudiantil ha aprendido a adaptarse a estas circunstancias $\mathrm{y}$ ha consolidado competencias para la vida en el plano personal y social.

La experiencia de enseñar a distancia de la población docente participante en el estudio significó aprender nuevas formas de enseñar; se apropiaron de conocimientos en el uso de la tecnología, en la implementación de estrategias didácticas diversificadas y en mecanismos de comunicación con la comunidad estudiantil y las familias; lo anterior condujo a innovar en su práctica pedagógica. Sin duda, transformaron su quehacer docente con la preparación de cuadernillos específicos al tomar como referencia las necesidades educativas del alumnado, diseñaron videos didácticos y explicativos, y usaron diversas plataformas para conjugar las actividades de los libros de texto gratuito, la programación televisiva y materiales didácticos propuestos por la SEP con el único objetivo de lograr el aprendizaje de la población escolar.

Sin embargo, incluso cuando el profesorado refleja nuevos aprendizajes que enriquecen su experiencia pedagógica en tiempos de la COVID-19, discutimos que la comunidad docente requiere apoyo prioritario en este proceso de confinamiento y para la inminente reapertura de las escuelas. Consideramos que se debe priorizar la formación, asesoría y acompañamiento para trabajar en formatos de educación con modalidad de enseñanza híbrida, incluida la formación en el uso educativo de herramientas y plataformas tecnológicas, así como en la selección de aprendizajes esperados o ajustes razonables y estrategias educativas que deberá implementar para atender la diversidad escolar que recibirá en el aula. Asimismo, se debe anticipar la formación docente en materia de habilidades socioemocionales para afrontar los efectos de pospandemia de la población estudiantil y su familia.

Finalmente, con fundamento en los resultados, exponemos algunas realidades, retos y aspiraciones que surgieron de las aportaciones de los docentes participantes: 


\section{Realidades}

- Esta pandemia puso de manifiesto que muchas familias no cuentan con recursos tecnológicos ni conexión a internet, lo que hace evidente la desigualdad social.

- No se identifica proceso formativo docente de la estrategia de educación a distancia "Aprende en Casa", impulsada por el gobierno federal, lo que origina que cada colectivo escolar organice a su entender y con recursos propios su enseñanza.

- Los padres y las madres de familia están sumergidos en la actividad laboral y tareas de casa y minimizan las acciones escolares de sus hijos e hijas.

- Hay docentes comprometidos que han transitado la capacitación o autocapacitación para enfrentar los retos pedagógicos y atender la diversidad escolar, y hay docentes con poca o nula información respecto a la situación escolar y familiar del alumnado.

- Los criterios de evaluación del aprendizaje son imprecisos por la falta de comunicación y participación del alumnado y su familia.

\section{Retos}

- Garantizar una educación inclusiva que priorice las oportunidades de aprendizaje y sostenga como eje rector la conectividad, los recursos tecnológicos, la adecuación de los contenidos al ámbito digital, así como las habilidades digitales del profesorado.

- Diseñar estrategias de acercamiento a hogares sin conectividad, viables y efectivas para lograr la razón de ser de la educación: el aprendizaje y la participación de la población infantil.

- Diseñar e implementar un plan de regularización para aquella población estudiantil que no tiene a su alcance la tecnología y la conectividad con la finalidad de recuperar los aprendizajes esperados.

- Establecer la formación académica del colectivo directivo y docentes en el uso de herramientas digitales de educación.

- Impulsar otro tipo de aprendizajes y otra forma de aprender, así como de enseñar; es decir, que las acciones vayan encaminadas al desarrollo de habilidades de autoaprendizaje y la función docente como facilitador más que transmisor de aprendizajes esperados.

- Impulsar la formación docente en modalidad híbrida para la adquisición de metodologías y estrategias diversas para la planificación de aprendizajes esperados.

\section{Aspiraciones}

- La oportunidad de acercar la escuela al contexto donde se desenvuelve el alumnado. La pandemia manifiesta la oportunidad de que el estudiante desarrolle competencias para la vida que impacten en el plano personal y social. 
- Impulsar una educación inclusiva, equitativa, pertinente y de calidad para minimizar o erradicar las barreras para el aprendizaje y la participación de la población estudiantil sin acceso a la tecnología y la conectividad.

- Dignificar la acción docente por varias razones: se han reinventado, innovado, autoformado; han trasladado el ámbito laboral a la intimidad de su hogar con jornada de trabajo extendido más allá de los límites saludables; han buscado alternativas para diversificar la enseñanza y, sobre todo, han soportado críticas sin sentido por parte de la sociedad sin conocer la responsablidad de la labor pedagógica que se realiza.

\section{REFERENCIAS BIBLIOGRÁFICAS}

Aguilar Gordón, F. del R. (2020). Del aprendizaje en escenarios presenciales al aprendizaje virtual en tiempos de pandemia. Estudios Pedagógicos, vol. 46, núm. 3, pp. 213-223. https://doi.org/10.4067/S0718-07052020000300213

Albalá Genol, M. Á. y Guido, J. I. (2020). La brecha socioeducativa derivada del Covid-19: posibles abordajes desde el marco de justicia. RLEE, nueva época, vol. L, número especial, pp. 173-194. https://doi.org/10.48102/rlee.2020.50. ESPECIAL.101

Centro de Investigación Avanzada en Educación, Universidad de Chile/Centro de Investigación para la Educación Inclusiva, Pontificia Universidad Católica de Valparaíso (2020). Covid-19. Nuevos contextos, nuevas demandas y experiencia docente en Chile. La enseñanza vivida por 2.205 docentes de establecimientos educacionales del país. Chile: CIAE/Unesco/EduGlobal.

CEPAL (2020). Universalizar el acceso a las tecnologías digitales para enfrentar los efectos del Covid-19. https://www.cepal.org/es/comunicados/cepalpropone-garantizar-universalizar-la-conectividad-asequibilidad-tecnologias

Coneval (2021). Caracterización y análisis del diseño de la estrategia Aprende en Casa. Ciudad de México. https://www.coneval.org.mx/Evaluacion/Documents/Informes/Caracterizacion_Aprende_Casa.pdf

Díaz-Barriga, Á. (2020). La escuela ausente, la necesidad de replantear su significado. En Educación y pandemia. Una visión académica. México: Instituto de Investigaciones sobre la Universidad y la Educación, UNAM.

Domínguez-Álvarez, B., López-Romero, L., Gómez-Fraguela, J. A. \& Romero, E. (2020). Emotion regulation skills in children during the Covid-19 pandemic: Influences on specific parenting and child adjustment. Revista de Psicología Clínica con Niños y Adolescentes, vol. 7, núm. 3, pp. 81-87. https://doi. org/10.21134/rpcna.2020.mon.2042

Espada, J. P., Orgilés, M., Piqueras, J. A. y Morales, A. (2020). Las buenas prácticas en la atención psicológica infanto-juvenil ante el Covid-19. Clínica y Salud, vol. 31, núm. 2, pp. 109-113.

Hernández Sampieri, R., Fernández Collado, C. y Baptista Lucio, M. del P. (2014). Metodología de la investigación (6⿳a ed). México: McGraw-Hill.

Hernández Vergel, V. K., Prada Núñez, R. y Hernández Suárez, C. A. (2021). Afectaciones ocupacionales y emocionales derivadas del aislamiento social en tiempos del Covid-19. Un estudio de casos. Revista Boletín Redipe, vol. 10, núm. 2, pp. 295-311. https://revista.redipe.org/index.php/1/article/view/1214 
SEP (2020). Anexo 1. Orientaciones para apoyar el estudio en casa de niñas, niños y adolescentes. Educación preescolar, primaria y secundaria. México.

Unesco (2020). El coronavirus covid-19 y la educación superior: impacto y recomendaciones. https://www.iesalc.unesco.org/2020/04/02/el-coronaviruscovid-19-y-la-educacion-superior-impacto-y-recomendaciones

Valcarce Fernández, M. (2011). De la escuela integradora a la escuela inclusiva. Revista Innovación Educativa, núm. 21, pp. 119-131. https://minerva.usc.es/xmlui/bitstream/handle/10347/6228/pg_121-134_in21_1. pdf? sequence $=1$ \&isAllowed $=y$ 